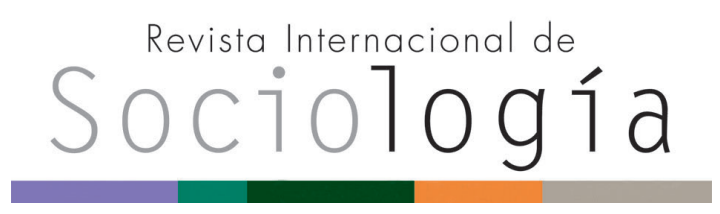

Revista Internacional de Sociología RIS vol. 73 (3), e019, septiembre-diciembre, 2015, ISSN-L:0034-9712

DOl: http://dx.doi.org/10.3989/ris.2013.04.08

\section{DETERMINANTES DEL SALARIO EN CHILE. Un análisis desde el paradigma de la reproducción social}

\author{
LUIS MIGUEL RODRIGO \\ Universidad Católica del Norte. Antofagasta. Chile \\ Irodrigo@ucn.cl
}

Cómo citar este artículo: Rodrigo, L.M. (2015), "Determinantes del salario en Chile. Un análisis desde el paradigma de la reproducción social", Revista Internacional de Sociología, 73, n³. Doi: http://dx.doi.org/10.3989/ris.2013.04.08

\section{Resumen}

Frente al paradigma económico neoclásico, hegemónico en la explicación del salario, se presenta el paradigma sociológico de la Reproducción Social, a partir de la obra de Pierre Bourdieu, para dar cuenta de la diferencia salarial en Chile. Después de exponer y comparar ambos paradigmas teóricos, se describe el caso de estudio, destacando las peculiaridades del modelo neoliberal chileno. Para comparar la capacidad explicativa de ambos enfoques, se estima el salario de los chilenos mediante la encuesta de Caracterización Socioeconómica Nacional 2009 (CASEN) a partir de dos modelos estadísticos, el modelo neoclásico basado en las ecuaciones de Mincer y un modelo que trata de capturar empíricamente los factores explicativos propios del paradigma de la Reproducción Social. Los resultados indican que, en el caso de Chile, el modelo Reproductivo tiene mayor capacidad explicativa que el Neoclásico, tanto para el conjunto de los asalariados, como para cada una de las generaciones que lo componen.

\section{Palabras Clave}

Bourdieu; Modelo Reproductivo; Sociología económica; Teoría Neoclásica.

\section{DETERMINANTS OF WAGES IN CHILE. An analysis from the paradigm of social reproduction}

Copyright: () 2015 CSIC. Este artículo de acceso abierto distribuido bajo los términos de la licencia Creative Commons Attribution-Non Commercial (by-cn) Spain 3.0.

Recibido: 08/04/2013. Aceptado: 03/03/2014

Publicación online: 24/08/2015

\section{AbstRact}

We use the sociological paradigm of Social Reproduction, proposed by the work of Pierre Bourdieu, to explain the wage gap in Chile, in opposition to the Neoclassical economic paradigm, currently hegemonic in the analysis of wage differentials. We expose and compare both theoretical paradigms and describe the case study highlighting the peculiarities of the Chilean neoliberal model. We estimate the salary of the Chilean population in order to explain the explanatory capacity of both theoretical approaches. We estimate two statistical models using the National Socioeconomic Characterization survey (CASEN 2009), firstly, the Neoclassical model based on Mincer equations and, secondly, a model that tries to empirically capture the explanatory factors specific to the paradigm of Social Reproduction. The results indicate that, in the case of Chile, the Reproductive model has greater explanatory power than the Neoclassical model, both for the whole group of workers, and for each of the generations of this group.

\section{KEYWORDS}

Bourdieu; Economic sociology; Neoclassical theory; Reproductive model, 


\section{INTRODUCCIÓN}

Desde los años sesenta del siglo pasado, la Teoría Neoclásica, paradigma dominante en Economía, ha atribuido las diferencias salariales a la productividad individual de los trabajadores, que estaría relacionada fundamentalmente con el nivel de educación. Desde entonces, la productividad individual se ha convertido en el principal eje explicativo para interpretar la diferencia de ingresos y el aumento del nivel educativo, en la principal estrategia política para reducir la desigualdad social.

Sin embargo, el análisis empírico muestra insistentemente que, en todas las sociedades, el salario está fuertemente relacionado con un conjunto de propiedades sociales, tales como el género, el grupo étnico, la raza, el origen social y el origen territorial. Aunque los estudios realizados desde el paradigma Neoclásico han tratado de incorporar algunas explicaciones ex post sobre los efectos de estas propiedades sociales, les resulta imposible articular una interpretación que no traicione su eje explicativo, esto es, la productividad individual. Posiblemente, una de las consecuencias de este problema narrativo sea la proliferación de los llamados trabajos "empíricos", que tratan de explicar $^{1}$ el salario a partir de una minería de datos ${ }^{2}$, sin aportar ninguna explicación (teórica) del fenómeno, es decir, sin construir un relato que dé cuenta de él.

Frente a esto proponemos estudiar los determinantes del salario desde el paradigma de la Reproducción Social, que suele ser utilizado en Sociología para explicar la trayectoria escolar o la posición social. Entendemos que este paradigma puede integrar la mayoría de la evidencia empírica en una explicación general y holística del fenómeno. En este sentido, nos distanciamos tanto del paradigma teórico hegemónico (el Neoclásico) como de los trabajos "empíricos" (ateóricos). Hay que señalar que en este artículo nos circunscribiremos a los determinantes sociales de la oferta de trabajo, es decir, a los relacionados con las características de los asalariados, excluyendo los determinantes de la demanda, esto es, de la estructura económica de los mercados (tamaño de empresas, sectores productivos, etc.).

El artículo se organiza en cinco apartados; el marco teórico donde se exponen el paradigma Neoclásico y el de la Reproducción Social, realizando un ejercicio comparativo entre ellos en el que se critican los supuestos teóricos neoclásicos; el caso de estudio donde se describen las características más relevantes del modelo neoliberal chileno; una revisión bibliográfica en la que se resumen los distintos trabajos que buscan explicar la diferencia salarial en Chile;

\footnotetext{
1 Entendido como la proporción de la varianza del salario que puede ser atribuida a las variables independientes.

2 Nos referimos a la búsqueda, sin hipótesis previas, de algún tipo patrón en las bases de datos.
}

el apartado metodológico donde se presenta la base de datos utilizada, las variables y la técnica estadística usadas; el apartado de resultados donde se compara empíricamente el modelo Neoclásico con el Reproductivo con el objeto de conocer sus niveles de adecuación al caso chileno; por último, en las conclusiones se sintetiza la crítica teórica de los paradigmas y las diferencias empíricas entre los modelos estadísticos, se sugieren nuevas variables que ayudarian a mejorar nuestro análisis y se proponen un conjunto de líneas de investigación complementarias.

\section{Marco teórico}

El paradigma Neoclásico, al menos desde Alfred Marshall, ha estado preocupado por los determinantes de la productividad. Sin embargo, su explicación contemporánea de la diferencia salarial, apareció en primer lugar con la Teoría del Capital Humano y posteriormente con la Teoría de la Señalización. Estos modelos son los más utilizados, habitualmente en forma complementaria, en los estudios empíricos.

La Teoría del Capital Humano, desarrollada por Schultz (1983) y Becker (1983) en los años sesenta, postula que los conocimientos que poseen las personas determinan su productividad individual $y$, por tanto, conforman una especie de capital que, al igual que la posesión de cualquier capital físico, puede ser rentabilizado en el mercado. Así, cuanto más conocimiento acumule un individuo más productivo será y recibirá por ello mayor ingreso. Por tanto, el núcleo de la Teoría del Capital Humano se basa en la relación causal conocimiento-productividad individual-salario.

Las formas de acumulación de conocimiento susceptible de ser rentabilizado en el futuro en forma de salarios son básicamente dos: la educación formal adquirida en el sistema educativo y la formación laboral adquirida en la empresa. La adquisición de estos conocimientos es presentada así como un tipo de inversión a largo plazo. Los individuos realizarían un análisis coste-beneficio donde evalúan el tiempo y el esfuerzo que requiere una determinada inversión educativa así como su costo de oportunidad, es decir, los ingresos que dejarían de percibir durante el proceso formativo. Como resultado de lo anterior, algunos individuos estarían dispuestos a renunciar a tener ingresos a corto plazo, con la expectativa de obtener mayores ingresos a largo plazo, aumentando así su capital humano. Mientras que otros individuos no estarian dispuestos a aplazar sus ingresos, renunciando con ello a aumentar dicho capital.

Habría que esperar hasta 1974 para que Mincer desarrollase la ecuación de ingresos que permitiría probar empíricamente los supuestos de la Teoría del Capital Humano. La ecuación de Mincer se generalizó rápidamente, utilizándose desde entonces tanto para estimar la rentabilidad de la educación en una sociedad determinada, 
como para explicar las diferencias de ingresos entre sus trabajadores. Su expresión más habitual es la siguiente:

$$
\ln S_{i}=\beta_{0}+\beta_{1} E D U C_{i}+\beta_{2} E X P_{i}+E X P_{i}^{2}+u_{i}
$$

Donde: $S$ es el salario del individuo i; EDUC es el número de años de educación formal recibida; $E X P_{\text {i son }}$

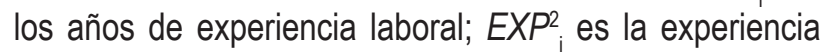
laboral al cuadrado; y $u_{i}$ es el término del error aleatorio que tiene una distribución normal con media 0 . Se asume por tanto que la educación formal tiene una relación lineal con el salario mientras que la experiencia laboral mantiene una relación cóncava, es decir, que aumenta el salario sólo hasta cierto punto, a partir del cual deja de afectarlo.

Posteriormente la ecuación de Mincer fue complejizada al ir incorporando las hipótesis de la Teoría de la Señalización, aunque se mantuvieron los factores explicativos (educación formal y experiencia laboral) que fundamentan la Teoría del Capital Humano.

La Teoría de la Señalización comenzó a configurarse una década más tarde, sin abandonar el paradigma Neoclásico, a partir de los trabajos de Spence, Stiglitz y Arrow. El denominador común de sus propuestas es que la educación formal no aumenta la productividad individual, sino que simplemente desvela (a las empresas) la capacidad innata de los trabajadores. En la Teoría de la Señalización tenemos que distinguir entre la hipótesis de la señalización y la hipótesis del filtro.

La hipótesis de señalización fue propuesta por Spence (1973) y Stiglitz (1975). Afirma que en un contexto de información imperfecta, los empresarios necesitan encontrar algún indicador de la productividad individual de los trabajadores antes de contratarlos, ya que ésta no es directamente observable, y conocerla después sería demasiado costoso. Entre las distintas características observables que presentan los trabajadores, el nivel educativo es la única que éstos pueden modificar invirtiendo en ella (a diferencia de la edad, la raza o el sexo), convirtiéndose por tanto en el mejor indicador (señal) de su productividad individual.

Por lo anterior, los futuros trabajadores tratarán de maximizar su nivel educativo, aunque sus resultados dependerán de sus habilidades innatas; es decir, los más capaces conseguirán alcanzar niveles superiores de educación. En este sentido, la educación no haría ganar productividad a los individuos, sino que más bien, los títulos escolares indicarían sus capacidades individuales. El salario que ofrece un empresario estaría ajustado, por tanto, a la valoración que realice, en términos de productividad, de las señales que emiten sus trabajadores. Dicha valoración podría variar tanto con el nivel educativo, como con el tipo de educación o con la institución que la imparte.

Por su parte, la hipótesis del filtro propuesta por Arrow (1973) afirma que, al igual que en el caso anterior, en un contexto de información imperfecta, el sistema educativo, especialmente la universidad, actúa como filtro clasificando a los estudiantes a partir de su capacidad individual. La universidad filtraría dos veces a los estudiantes, en un primer momento seleccionando a los alumnos que ingresan, ya que no todos son capaces de acceder a ella, y en un segundo momento, seleccionado a los titulados, porque no todos los estudiantes que ingresan consiguen egresar. Al igual que la anterior, esta hipótesis asume una relación directa entre éxito escolar y productividad laboral.

Para la comprobación empírica de la Teoría de la Señalización, se ha seguido utilizando, aunque modificada, la ecuación de Mincer. Los principales cambios en la ecuación de ingresos supusieron abandonar los supuestos de homogeneidad de la educación, ya que para Mincer no existía diferencia alguna entre los distintos tipos de educación o entre las instituciones que la ofrecen; y abandonar el supuesto de linealidad entre educación e ingreso, pues todos los años de educación aportaban el mismo retorno económico. Así, las nuevas ecuaciones de ingresos adaptadas a la Teoría de la Señalización, estiman la rentabilidad para distintos itinerarios educativos, para distintas instituciones y, sobre todo, para los distintos años de educación, distinguiendo entre los individuos que lograron titularse y los que no. Dichas modificaciones consiguieron capturar mejor el efecto de la educación en los salarios, aunque los factores explicativos continuaron siendo los mismos, la educación formal y la experiencia laboral.

No tardaron en aparecer las críticas a las explicaciones neoclásicas del salario, especialmente a la Teoría del Capital Humano, por parte de economistas norteamericanos pertenecientes a otros paradigmas teóricos (Perla 2007; Toharia 2005). Uno de sus principales críticos (Thurow 1983), responsable de la Teoría de la Competencia por los Puesto de Trabajo, demostró que los años de escolaridad no podían explicar las diferencias salariales, proponiendo que éstas se debían a la propia dinámica del mercado laboral. También pudo comprobar que la fuerte expansión educativa que se produjo en EEUU después del periodo de posguerra, no había producido una reducción de la desigualdad del ingreso y, finalmente, que el incremento de productividad que se había producido en ese periodo, no estaba relacionado con el aumento educativo de los trabajadores. Este aumento educativo, en vez de generar productividad y convergencia salarial, estaba produciendo sobrecualificación y subempleo. Junto con lo anterior, los trabajos empíricos sobre el mercado laboral, mostraban persistentemente que había factores extraeconómicos, como el género y la raza, que predecían mejor el salario que la educación (Perla 2007).

Además, no podemos dejar de observar que los modelos empíricos desarrollados por la Teoría Neoclásica para explicar el salario eluden la explicación del nivel educativo que aparece como un factor exógeno. Se deja por tanto sin comprobación empírica las (muy cuestionables) explicaciones 
neoclásicas sobre el nivel educativo que alcanzan los individuos. Recordemos que para la Teoría del Capital Humano la decisión de invertir o no en educación se debe a las preferencias (suponemos que innatas) de los individuos. Los más pacientes y racionales invertirán y los más impacientes o irracionales no lo harán. Para la Teoría de la Señalización, por su parte, todos los individuos intentarían alcanzar el nivel más alto de educación posible, por lo que sus resultados dependerán de sus capacidades innatas. Si bien es cierto que el paradigma Neoclásico, en su versión más ortodoxa, no se preocupa por el realismo de los supuestos, hay que señalar que, al evitarse su comprobación empírica, se renuncia a conocer su grado de veracidad, por lo que terminan convertidos en explicaciones axiomáticas.

Frente al paradigma económico Neoclásico, proponemos el paradigma sociológico de la Reproducción Social ${ }^{3}$ para explicar las diferencias salariales, en concreto nos centraremos en la teoría de Bourdieu (2008, 2003 y 2001) por considerarla la más adecuada para nuestra problemática. En su forma general, el paradigma de la Reproducción Social se preocupa por analizar (y denunciar) los mecanismos mediante los cuales se reproduce una estructura social determinada, en especial, las relaciones de dominación y explotación entre géneros, clases sociales, grupos raciales/étnicos y territorios (países o regiones).

Podemos deducir de la teoría bourdieuniana la siguiente tesis: la desigualdad social entre las familias (sus condiciones de existencia) determinará las trayectorias escolares de sus descendientes, generando así una nueva desigualdad (individual/escolar) que reproducirá a la anterior (social/familiar). Posteriormente, ambas desigualdades (social e individual) determinarán la trayectoria socio-laboral de los descendientes. En este proceso reproductivo, el sistema escolar tendrá un papel fundamental como legitimador de la desigualdad socioeconómica.

Para Bourdieu, las condiciones de existencia de un agente están determinadas por el conjunto de posiciones objetivas que ocupa en las distintas relaciones sociales. Así, además de conocer su posición en las relaciones de género y de raza/etnia, habría que saber su posición social y territorial de origen (familiar).

La posición social de una familia está determinada por la clase y el campo social al que pertenece. La clase social hace referencia al volumen global de capital con el que cuenta cada familia, así como a la estructura de dicho capital (peso relativo de cada tipo de capital). Para Bourdieu existen cuatro tipos de capital, el económico, el cultural, el social y el simbólico (estatus).

\footnotetext{
3 El origen de este paradigma podría situarse en el análisis marxista sobre la reproducción del sistema capitalista (Marx 2005; Marx y Engels 1994). Sin embargo, el ascendiente directo de la obra de Bourdieu es Althusser (1974) quién desarrollo una teoría centrada específicamente en la reproducción social.
}

Los tipos de capital familiar (Bourdieu 2001) determinantes para la trayectoria escolar de los estudiantes son los capitales cultural y económico, mientras que el capital social y el simbólico serán más importantes a la hora de rentabilizar el título académico en el mercado laboral.

El capital económico familiar está directamente relacionado con la capacidad de inversión escolar, las familias más ricas pueden permitirse trayectorias escolares más largas y caras, pudiendo acceder a instituciones más prestigiosas y alejadas. Por lo anterior, la importancia del capital económico para la trayectoria escolar es directamente proporcional a la desigualdad de un sistema educativo determinado.

Por su parte, el capital cultural consiste en la posesión de una cantidad de conocimientos y habilidades socialmente reconocidos y valorados. Dicho capital se puede encontrar de forma incorporada o institucionalizada. En su forma incorporada, supone la acumulación de cultura mediante su interiorización biológica y el proceso de adquisición se produce principalmente en el seno familiar (Bourdieu 2001: 143). El capital cultural incorporado está desigualmente distribuido entre clases sociales y territorios $^{4}$. A diferencia de éste, el capital cultural institucionalizado es un capital cultural objetivado por un reconocimiento legal en forma de título. Por lo tanto el valor obtenido con éste es independiente de su portador, aportando el mismo reconocimiento a todos los agentes que lo posean (Bourdieu 2000: 148). El objetivo de la inversión escolar es la obtención de este capital cultural institucionalizado, que podrá ser convertido en capital económico en el mercado laboral. Los estudiantes cuyos padres poseen una gran cantidad de capital cultural, lo adquirirán en el ámbito familiar y no sólo en el sistema escolar, condición que multiplicará su acumulación.

Finalmente, el capital social se refiere a una red duradera de relaciones de obligación mutua que potencialmente da acceso a sus participantes a todo el capital del grupo de agentes que la constituyen. Cuando los estudiantes concluyan su trayectoria escolar e ingresen al mercado laboral, el volumen de capital social de sus familias determinará en gran medida la rentabilidad socioeconómica de su título académico. Así, las familias con alto capital social lo podrán utilizar para que sus hijos consigan puestos elevados en la jerarquía ocupacional, mientras que los hijos de las familias más pobres en capital social, teniendo las mismas titulaciones, ocuparán posiciones inferiores.

La posición social de las familias también está determinada por el campo social que ocupan (Bourdieu 2008). Los campos son espacios sociales estructurados a partir de un conjunto de relaciones que se dan en su seno, que gozan de una autonomía relativa y de una lógica de funcionamiento

\footnotetext{
4 Entre municipios urbanos y rurales, pero también entre regiones periféricas y la metrópoli.
} 
propia. Así podemos hablar del campo escolar, del económico, del político o del artístico. Cada campo social permite la acumulación de un tipo de capital específico, por lo que determina la estructura del capital familiar, afectando así a la trayectoria social de sus descendientes. Las familias, además, tienen más información del campo al que pertenecen, pudiendo así orientar a sus hijos para que ingresen en él (si es provechoso) o para que se alejen de él (si está en decadencia). Por otro lado, las familias consiguen acumular con los años un conjunto de capitales específicos de su campo social, que sus descendientes sólo podrán aprovechar si ingresan en él.

Como decíamos anteriormente, para Bourdieu, el sistema escolar consigue, mediante una serie de operaciones (Bourdieu y Passeron 2001), transformar una parte del capital familiar (especialmente su capital cultural) en el capital escolar ${ }^{5}$ de los hijos, reproduciendo así la desigualdad social inicial. A partir de sus propiedades sociales (clase, género, raza/etnia) el sistema escolar dirigirá a los estudiantes hacia itinerarios educativos de desigual valor social. Al final, los efectos del sistema escolar son totalmente diferentes para los distintos grupos sociales. Las

5 El capital escolar es el subtipo de capital cultural que es reconocido, administrado y valorado por el sistema escolar. clases dominantes, al permanecer más tiempo en el sistema escolar, elegir los itinerarios más valorados y los centros más prestigiosos, logran acumular una gran cantidad de capital escolar, que utilizarán para adquirir y legitimar una posición superior en la estructura ocupacional. Para las clases dominadas, con una permanencia menor, elección de itinerarios más desvalorados y de centros menos prestigiosos, el paso por el sistema escolar no les servirá para conseguir posiciones elevadas en la estructura ocupacional, pero sí para conocer y reconocer la posesión de capital escolar como el principio legítimo de desigualdad social y para reconocer a la cultura de la clase dominante como "cultura superior".

Respecto a la socialización de género, esta continúa reproduciendo la matriz patriarcal generando dos subjetividades bien diferenciadas, la masculina y la femenina, asociadas a sus correspondientes roles de género (Bourdieu 2003). En lo referente al trabajo, la división sexual que realiza el patriarcado supone preparar a los hombres para la producción social, mientras que las mujeres son preparadas para realizar el trabajo de reproducir las fuerzas productivas, lo que supone no sólo la reproducción biológica sino también la social. Los trabajos femeninos, no solo son diferentes a los masculinos, sino que tienen una menor valoración social y, por lo tanto, generan menos ingresos.

Cuadro 1.

Las operaciones de producción y reproducción

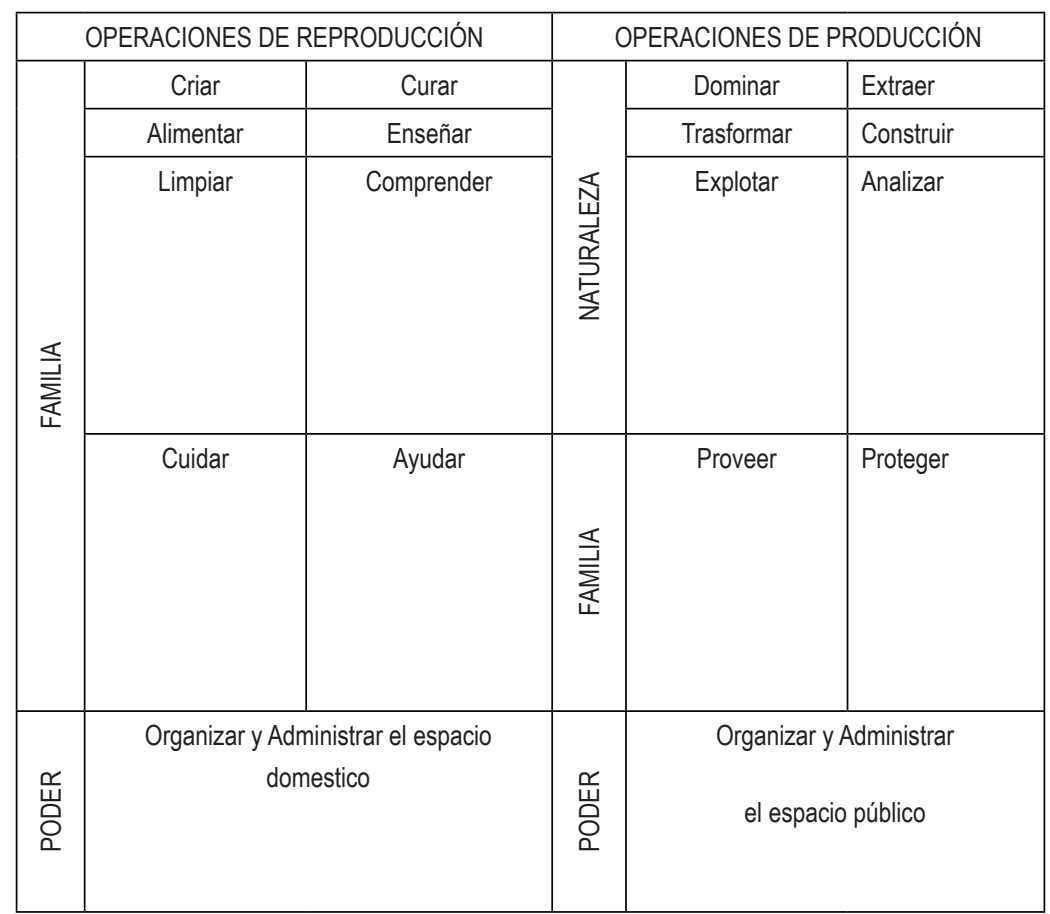

Fuente: Elaboración propia a partir de Bourdieu (2003) 
Resumiendo, podemos decir que las condiciones de existencia de los agentes, es decir, aquellas propiedades sociales que adquirirán nada más nacer (género, raza/etnia y posición social y territorial de origen), determinarán en un primer momento biográfico su trayectoria escolar y, en un segundo momento, la interacción entre ambas determinará su trayectoria socio-laboral y en definitiva, su posición social de destino.

Primer momento biográfico: CONDICIONES DE EXISTENCIA (CE) $\Rightarrow$ TRAYECTORIAESCOLAR (TE) Segundo momento biográfico: CE * TE $\Rightarrow$ POSICIÓN SOCIAL DE DESTINO (Salario et al. ${ }^{6}$ )

Podemos realizar una comparación entre estos dos paradigmas teóricos a partir de cuatro criterios diferenciadores: el objeto/sujeto de análisis; la posición epistemológica; el eje explicativo; y la posición política:

- En primer lugar observamos que el objeto/sujeto de análisis del paradigma Neoclásico siempre es un individuo abstracto (asocial y ahistórico), mientras que para el paradigma de la Reproducción Social son distintos grupos sociohistóricos: la familia, la clase social, el género o las comunidades étnicas.

- En segundo lugar podemos apreciar cómo el paradigma Neoclásico presenta una epistemología particularista, al preocuparse exclusivamente por la relación causal entre dos eventos (educación y salario) que quedan así aislados del resto del universo social. Frente a este, el paradigma de la Reproducción Social presenta una epistemología holística, típicamente sociológica, al analizar un

6 La posición social está compuesta por la clase y el campo social que ocupará el agente. La clase social estará conformada, entre otros, por su capital económico, del cual el salario es una parte. hecho social determinado (salario) a partir de la totalidad de relaciones sociales que lo sobredeterminan.

- En tercer lugar, podemos afirmar que el eje explicativo del paradigma Neoclásico es la productividad individual, ya sea esta innata, como afirma la Teoría de la Señalización, o adquirida, como propone la Teoría del Capital Humano. En ambos casos será la productividad individual la que determinará los ingresos de los individuos. A partir de este supuesto, se deduce necesariamente que las diferencias salariales que existen entre distintos grupos sociales se deben a su diferente productividad. Esto nos llevaría a conclusiones absurdas, como que las mujeres, los negros/indígenas y las clases populares serían menos productivos que los hombres, los blancos y las clases superiores, ya fuese porque tienen una menor capacidad innata (Teoría de la Señalización) o porque son más impacientes e irracionales al no haber decido invertir en educación (Teoría del Capital Humano). Por su parte, el paradigma de la Reproducción Social utiliza como eje explicativo la herencia social, es decir, la hipótesis de que a partir de una serie de procesos, la estructura social consigue reproducirse intergeneracionalmente. Por tanto, las diferencias salariales entre los grupos sociales a los que hicimos referencia estarían explicadas por las posiciones históricas de dichos grupos en la estructura social y no por la capacidad individual de sus miembros.

- Finalmente podemos afirmar que el paradigma Neoclásico mantiene una posición política conservadora, pues legitima al orden social al explicar las diferencias salariales a partir de un discurso meritocrático que oculta las relaciones sociales en las que están insertos los agentes. Frente a él, el paradigma de la Reproducción Social se sitúa en una posición política crítica, al denunciar que las causas de la desigualdad social son estructurales.

Cuadro 2.

Comparación entre los dos paradigmas

\begin{tabular}{|c|c|c|}
\hline CRITERIOS DIFERENCIADORES & PARADIGMA NEOCLÁSICO & $\begin{array}{c}\text { PARADIGMA DE LA REPRODUCCIÓN } \\
\text { SOCIAL }\end{array}$ \\
\hline Objeto/sujeto de análisis: & Individuo abstracto & Grupos sociohistóricos \\
\hline Posición epistemológica: & Particularista & Holística \\
\hline Eje explicativo: & Productividad individual & Herencia social \\
\hline Posición política: & Conservadora & Crítica \\
\hline
\end{tabular}

Fuente: Elaboración propia. 


\section{Caso de estudio}

Consideramos que el caso chileno resulta especialmente pertinente para el estudio de los determinantes del salario, pues el país aún mantiene vigente el modelo socioeconómico neoliberal que impuso la dictadura militar (1973-1990) bajo la directa supervisión de Milton Friedman, resultando difícil encontrar un ejemplo mejor del modelo neoliberal que propuso la Escuela de Chicago (M. Garretón y R. Garretón 2010; Camargo 2007; Canales 2007; Lagos 2007). En este sentido, al haberse reducido drásticamente tanto el tamaño como la intervención del Estado, la teoría Neoclásica debería tener mayor capacidad explicativa que cualquier otra.

La primera característica que podemos señalar del modelo neoliberal chileno, dejando a un lado los atentados contra los Derechos Humanos que requirió su puesta en marcha, es el fuerte incremento que produjo en la desigualdad social, que se ha mantenido, sorprendentemente, durante los gobiernos democráticos. Chile es actualmente uno de los países con mayor desigualdad socioeconómica del mundo (coeficiente de Gini de 0,53), por encima de países como Nigeria (CASEN 2009).

Otra característica del modelo fue la instalación de un conjunto de ideas-fuerza (Moulian 2002). Una de ellas fue la del rechazo total a cualquier paradigma socioeconómico distinto al Neoclásico, concretamente a la versión monetarista creada por Friedman en la Escuela de Chicago. Este paradigma pasó a convertirse en la teoría socioeconómica oficial, acusando a cualquier otro de acientífico e irracional. El paradigma Neoclásico recibió un fuerte apoyo internacional con la llegada al poder de Thatcher y Reagan y desde diferentes organismos como el Banco Mundial, el Fondo Monetario Internacional y la Organización Mundial del Comercio. Por otro lado, el fuerte crecimiento económico experimentado por Chile en el quinquenio 1977-1982 y en el decenio 1987-1997 terminó por legitimarlo "técnicamente".
Desde la hegemonía del paradigma Neoclásico se rediseñó toda la institucionalidad social con la intención declarada de crear un conjunto de mercados (de salud, de pensiones y educativo entre otros) eficientes y competitivos. El sistema educativo fue rediseñado en la reforma de 1980, lo que supuso pasar de un modelo público administrado por el Estado a un modelo descentralizado administrado por los municipios, mediante una lógica de libre mercado. La reforma favoreció la aparición de 34 universidades privadas, que junto a las 9 anteriores a 1981 suponen el $73 \%$ de las universidades chilenas. Existen 16 universidades estatales; aunque el precio de sus matrículas no es inferior al de las privadas, de hecho, el aporte del Estado supone sólo el 17\% de sus ingresos, el $34 \%$ corresponde a matrículas y $49 \%$ son recursos autogenerados (OCDE 2009). Las universidades más prestigiosas, son justamente las anteriores a la reforma (1981), llamadas universidades tradicionales.

Los principales resultados de modelo educativo fueron: una fuerte segregación social de los alumnos a partir de los diferentes precios/calidades ofertados por el mercado educativo; y un contundente aumento de matriculados en la educación superior, financiados fundamentalmente con gasto familiar.

El efecto segregador del modelo educativo chileno puede apreciarse en el gráfico 1. Como podemos observar existe una gran desigualdad social entre los estudiantes pertenecientes a las escuelas municipales (públicas), subvencionadas, y privadas. Además, esta desigualdad social se reproduce nítidamente en sus resultados escolares (Puntajes PISA), especialmente en la Prueba de Selección Universitaria (PSU), que permite el acceso y la selección de universidad.

Por este motivo, organismos internacionales como la Organización para la Cooperación y el Desarrollo Económico (OCDE) han solicitado reformas tendentes a disminuir la desigualdad de acceso de los estudiantes pertenecientes a grupos de bajos ingresos (OCDE 2009).

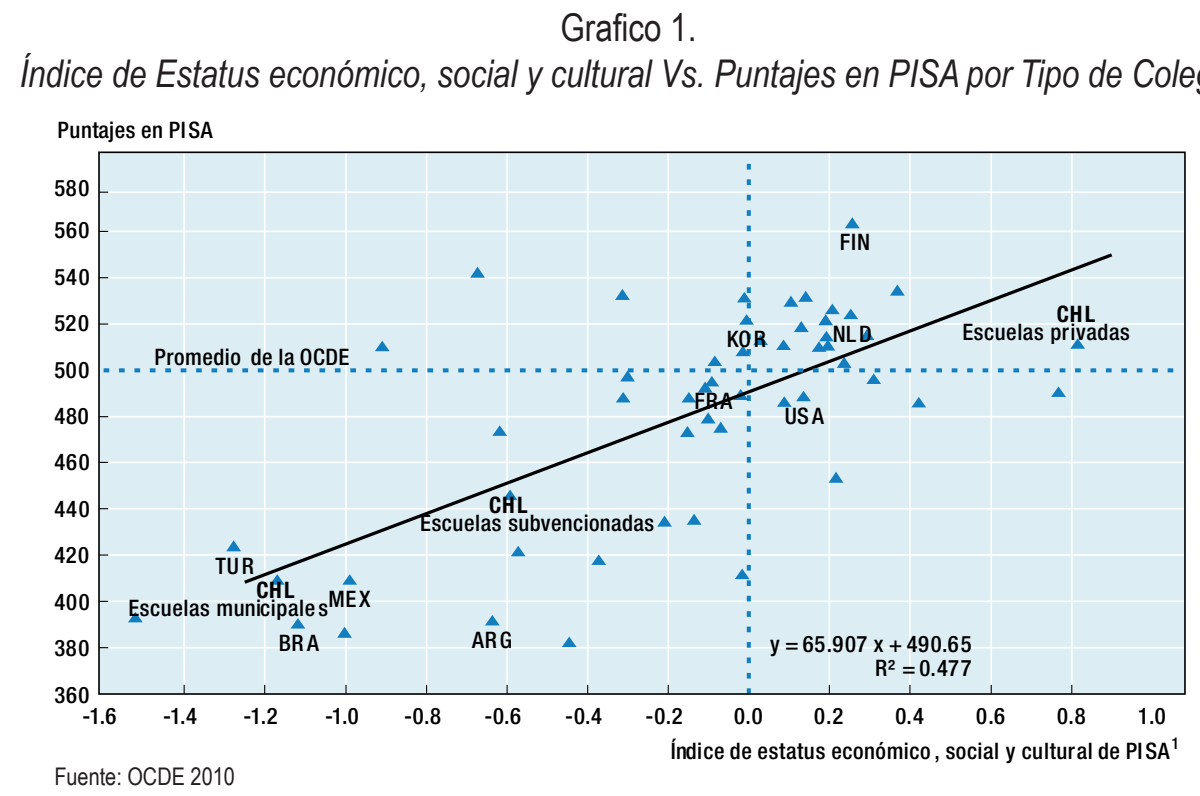


Gráfico 2.

Escolaridad por percentiles y cohortes de edad

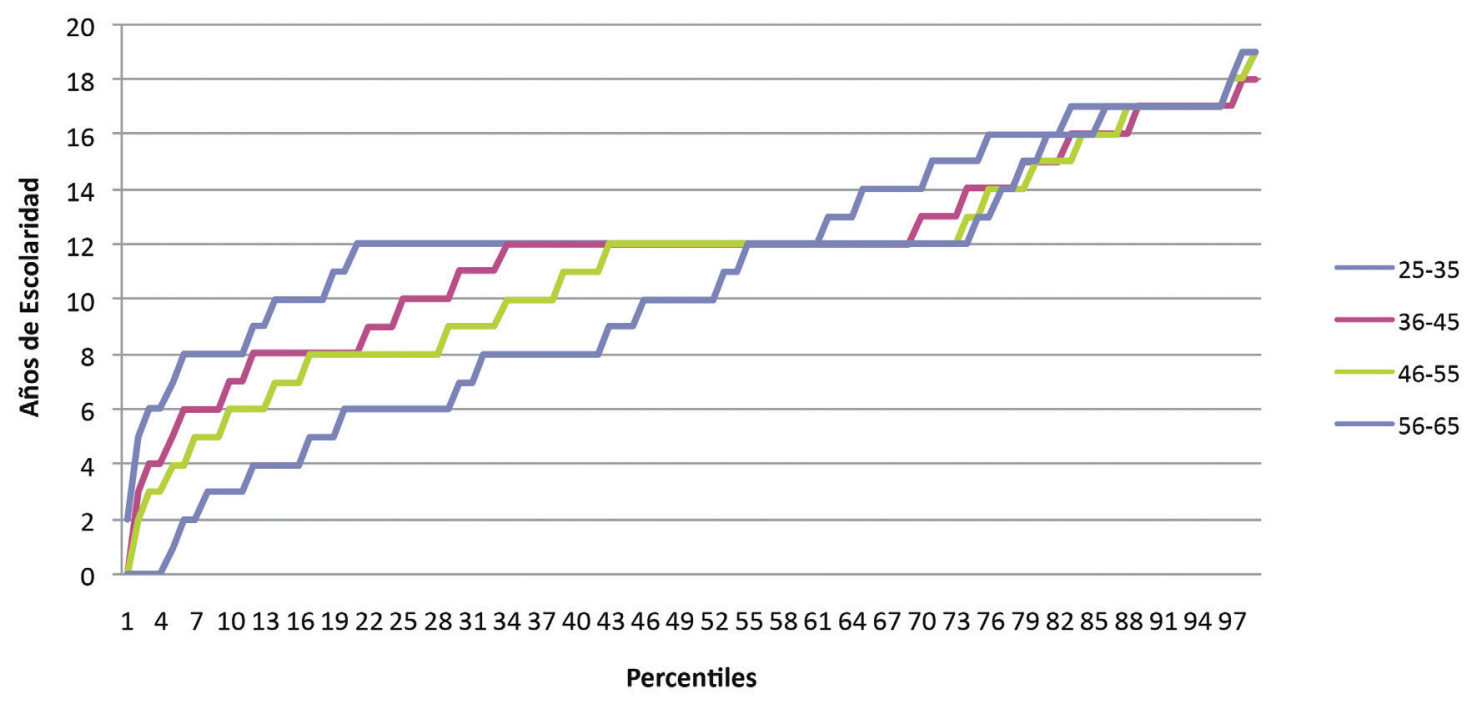

Elaboración propia a partir de CASEN 2009

Por otra parte, el importante aumento en la extensión de la educación superior ha sido señalado en múltiples trabajos (Castillo y Cabezas 2010; Brunner 2009a y 2009b; OCDE 2009; Zapata y Tejeda 2008). Asimismo, el informe del Consejo asesor presidencial para la educación (CAP) indica que la proporción de jóvenes de 18 a 24 años pertenecientes al I y || quintil que ingresaron a la educación superior se ha duplicado entre 1998 y el 2006. Este informe también destaca que cerca del $70 \%$ de quienes asisten hoy a una institución de Educación Superior en Chile, son hijos de padres que nunca asistieron a ese nivel educacional (CAP, 2008). En el gráfico 2 podemos observar cómo efectivamente, se ha producido un aumento de los años de escolaridad en las cohortes más jóvenes. Comprobamos cómo, por ejemplo, sólo el $21 \%$ de la población de 25 a
35 años tiene menos de 12 años de escolaridad, mientras que esta cifra aumenta hasta el $55 \%$ para la población de 56 a 65 años.

Este fuerte incremento del nivel educativo debería producir, según la Teoría del Capital Humano, un incremento en los ingresos de las generaciones más educadas, debido a una mayor productividad. También sería esperable que en estas generaciones se redujese la desigualdad económica, si es que esta se debe, fundamentalmente, a diferencias educativas. Por lo anterior nos deberían resultan paradójicos los resultados que mostramos en el gráfico 3. Como se puede observar, las cohortes más jóvenes no obtienen mayores ingresos, pese a tener un nivel significativamente más alto de escolaridad. Aunque quizá lo más sorprendente sea comprobar que las cuatro cohortes

Gráfico 3.

Ingreso por percentiles y cohortes de edad.

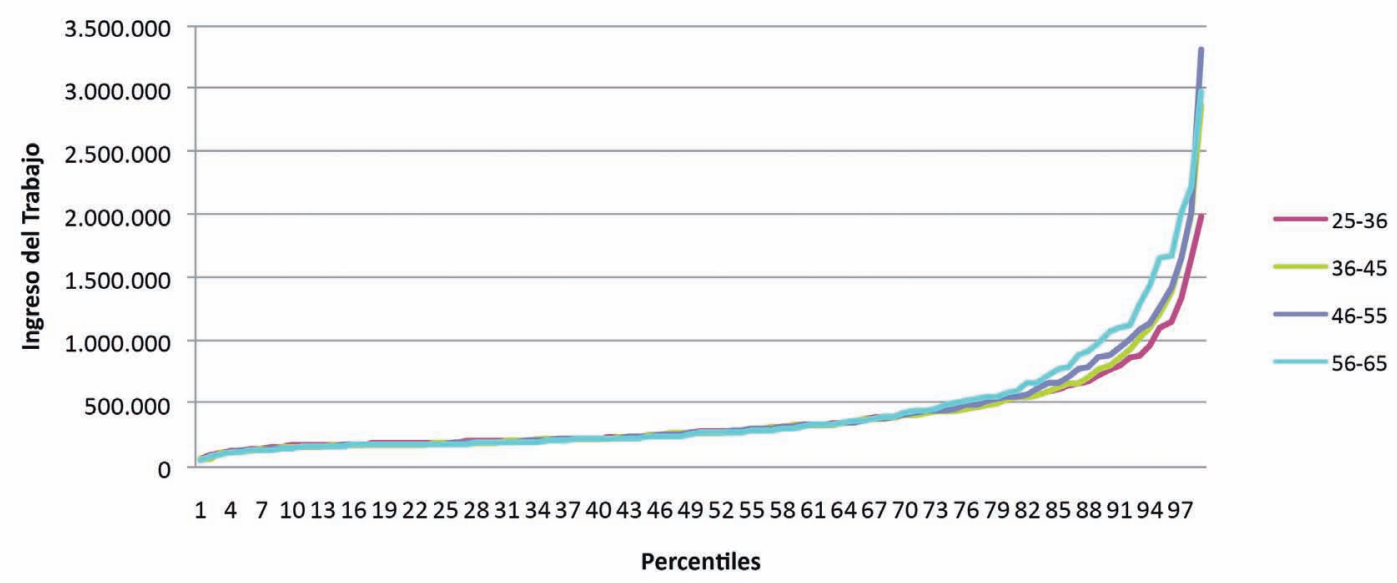

Elaboración propia a partir de CASEN 2009 
comparadas mantienen una desigualdad de ingreso similar, pese a que las más jóvenes han reducido de forma importante su desigualdad educativa.

¿Por qué el aumento educativo de las generaciones más jóvenes no les genera mayores salarios? $Y$, sobre todo, ¿por qué la reducción de la desigualdad educativa no se traduce en una reducción de la desigualdad económica? Las familias chilenas han realizado en los últimos treinta años un esfuerzo educativo sin precedentes, sin embargo, los resultados prometidos por el modelo neoliberal no se han materializado. Puede que aún falten unas décadas para que se produzcan los resultados esperados, pero también es posible que, en contra de lo que afirma la Teoría del Capital Humano, las diferencias salariales no se deban ni exclusiva ni principalmente a las diferencias educativas y por tanto la reducción de estas, no afecte significativamente a la desigualdad económica.

\section{Revisión bibliográfica}

La bibliografía de la última década presenta un conjunto de trabajos empíricos sobre los determinantes del ingreso en Chile, unos realizados desde el paradigma Neoclásico (Sapelli 2009 y 2003; Ramos et al. 2009) y otros fuera de él (Núñez y Pérez 2007; Núñez y Risco 2004; Núñez y Gutiérrez 2004) aunque carentes de otro referente teórico. A continuación realizaremos una breve exposición de estos trabajos.

Desde dentro del paradigma Neoclásico habría que señalar los dos trabajos de Sapelli (2009 y 2003). En el primero de ellos, el autor estima las ecuaciones de ingreso para los años 1990 y 1998 utilizando las encuestas de la CASEN. Partiendo de la ecuación Mincer, el autor des- agrega progresivamente el nivel de escolaridad, identificando en sus resultados la existencia de un fuerte efecto titulación $n^{7}$ como anticipaba la Teoría de la Señalización, es decir, un importante aumento de ingresos en los años en que se completa un ciclo escolar y se obtiene un título académico. Sin embargo, también existe aumento del ingreso, aunque menor, en los años de escolaridad previos a la titulación, lo que no podría ser explicado por esta teoría, por ello concluye que la Teoría del Capital Humano y la Teoría de la Señalización son complementarías. Por otro lado, comparando los ingresos obtenidos por la educación en los años 1990 y 1998, concluye que la rentabilidad de la educación aumentó en ese periodo.

En su segundo trabajo sobre el tema, Sapelli (2009) pone a prueba dos metodologías diferentes para estimar el retorno salarial de la educación. En primer lugar estima la ecuación Mincer utilizando, como suele hacerse, un corte transversal a partir de las encuestas CASEN de los años 1990 y 2006 . Después, realiza las mismas estimaciones pero dividiendo la muestra por cohortes sintéticas. Para este segundo método, utiliza las Encuestas de Ocupación del Gran Santiago para los años 1957 a 2000 y la encuesta CASEN para los años 1990 a 2006. El autor concluye, en primer lugar, que la ecuación de Mincer (corte transversal) subestima el retorno salarial de la educación, ya que este aumenta cuando se utilizan cohortes sintéticas. $Y$ en segundo lugar, que el retorno salarial de los distintos niveles educativos está en proceso de convergencia.

7 En inglés sheepskin effect (efecto piel de cordero), en referencia al material con el que se confeccionaban los títulos académicos.

Gráfico 4.

Retornos de los distintos niveles de educación (por generaciones)

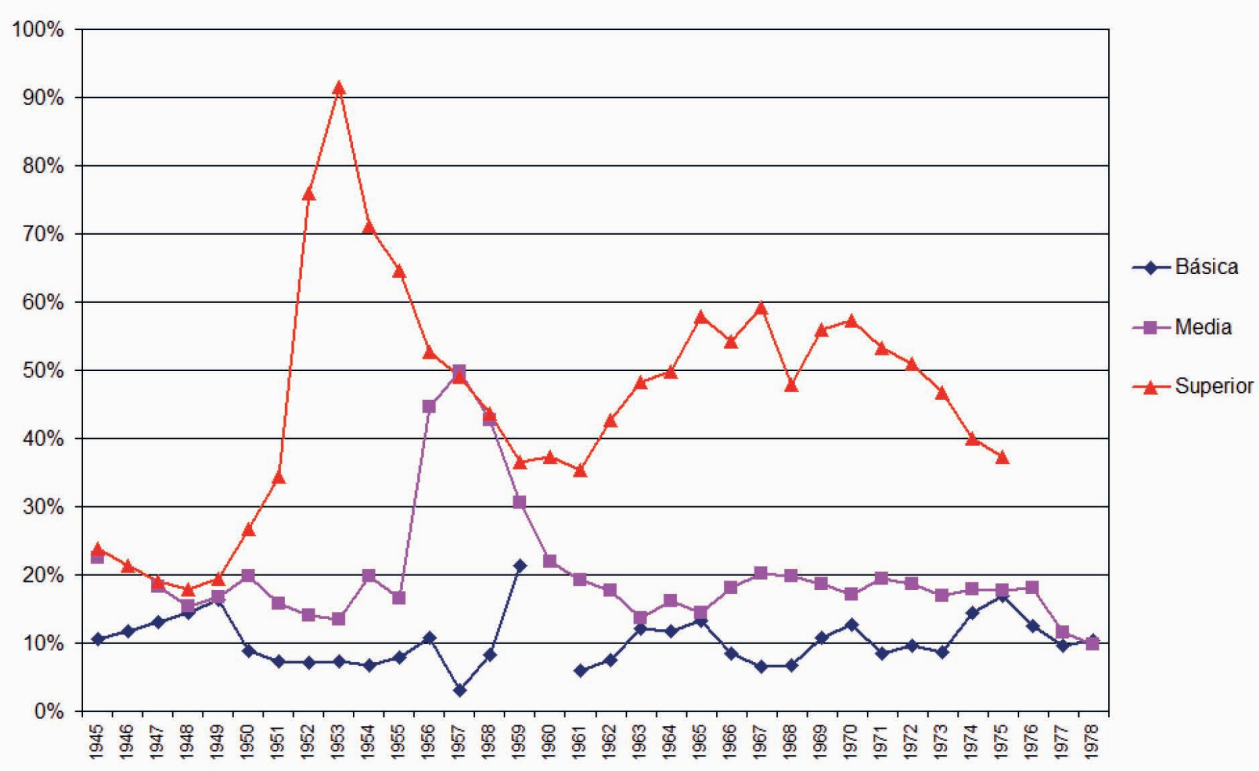

Fuente: Sapelli, 2009 pp. 19 
Como se puede apreciar en el gráfico 4, la educación básica siempre ha mantenido una tasa de retorno salarial próxima al 10\%. La educación media por su parte, mantuvo un retorno salarial del $20 \%$ para las generaciones nacidas entre 1960 y 1976. Sin embargo, para las dos últimas generaciones estudiadas (1977 y 1978), la tasa de retorno bajó hasta el 10\%, igualándose con la educación básica. Finalmente, el retorno de la educación universitaria presenta un descenso progresivo desde la generación de 1970 , para la que tuvo un retorno cercano al $60 \%$, hasta la generación de 1975, donde el retorno ya estuvo por debajo del $40 \%$. Si bien es cierto que, como afirma Sapelli, el retorno salarial de los distintos niveles educativos está convergiendo, lo está haciendo a la baja, es decir, la rentabilidad salarial de la inversión educativa es cada vez menor.

Sin abandonar el paradigma Neoclásico, el trabajo de Ramos et al. (2009) intenta determinar los factores que explican el salario de los egresados universitarios de las carreras de Ingeniería Comercial y de Contador Auditor. Para ello, utilizaron la base de datos del Departamento de Evaluación, Medición y Registro de la Universidad de Chile y la de un importante portal web de búsqueda de empleo. En primer lugar, estimaron la ecuación de ingresos de Mincer usando las variables de género, experiencia y años de educación. Posteriormente realizaron tres nuevas regresiones, a las que añaden las variables de estado civil, tamaño de la región, nivel educativo del padre, experiencia laboral auto-reportada, nivel de estudios, tipo de colegio, ranking en la educación media y en PSU (prueba de selección universitaria) y sector en que se trabaja. Los autores concluyen que la ecuación de Mincer es insuficiente para explicar las diferencias salariales de estos profesionales y defienden un modelo que incorpore otras dimensiones como el capital social familiar, la titulación conseguida y la capacidad individual.

Fuera ya del paradigma Neoclásico debemos hacer referencia a la interesante línea de trabajo de Núñez y sus colaboradores. Nos centraremos en tres estudios en los que se identifican distintos determinantes sociales del ingreso en Chile. En el primero de estos trabajos (Núñez y Gutiérrez 2004) los autores estiman las diferencias salariales entre los egresados de una de las principales universidades de Chile ${ }^{8}$ a partir de su procedencia socioeconómica. Sus resultados muestran que los egresados provenientes de los estratos socioeconómicos más altos tienen un salario un $50 \%$ mayor que aquellos que provienen de los estratos inferiores. Esta brecha salarial se mantiene después de controlar por las variables habituales que podrían influir en el salario. Por ello, los autores concluyen que el origen socioeconómico de los egresados afecta a su salario a partir de algún tipo de discriminación de los

\footnotetext{
8 Si bien no lo explicitan, entendemos que se refieren a la Universidad de Chile, ya que es la institución a la que pertenecen.
}

empleadores, siendo dicha diferencia mayor que la que se atribuye en la literatura a la raza o al género.

En el segundo trabajo (Núñez y Risco 2004) se estudia la movilidad intergeneracional del ingreso en la zona urbana del Gran Santiago utilizando la Encuesta de Ocupación y Desocupación del Gran Santiago del año 2004. Los resultados del estudio muestran que, comparado con otros países, Chile presenta un nivel muy bajo de movilidad intergeneracional del ingreso, siendo similar al caso brasileño. También se percibe un aumento de la movilidad del ingreso a partir de 1990, que los autores relacionan con el fuerte incremento de la escolaridad que experimentó el país.

En el último trabajo al que haremos referencia (Núñez y Pérez 2007), los autores estudian el efecto del apellido de los egresados de Ingeniería Comercial en la percepción que otros estudiantes tienen de su posición socioeconómica y la capacidad de dicha percepción para predecir la posición socioeconómica de estos egresados. Los casos se extraen de la base de datos de una importante universidad chilena y se realiza un experimento en el que unos grupos de estudiantes tienen que atribuir un estrato socioeconómico a estos egresados a partir de sus apellidos. Los resultados muestran que el apellido de los egresados explica la percepción que el resto de estudiantes tienen de su posición socioeconómica (posición subjetiva) y que dicha percepción explica una parte significativa del estrato socioeconómico de estos egresados (posición objetiva). Los autores concluyen que en Chile sigue existiendo una importante relación entre ascendencia familiar (apellidos) y estrato socioeconómico debido a la baja movilidad social que lo ha caracterizado históricamente.

En resumen, la literatura chilena sobre determinantes del ingreso se puede clasificar en tres tipos de estudios. En primer lugar tenemos los trabajos que desde el paradigma Neoclásico tratan de mejorar sus indicadores para capturar mejor los factores teóricos. Así por ejemplo, Sapelli (2009 y 2003) utiliza ecuaciones del ingreso empíricamente más sensibles que la de Mincer sin abandonar la explicación neoclásica.

En segundo lugar encontramos trabajos que partiendo de la ecuación de Mincer van agregando variables pertenecientes a dimensiones no relacionadas con el paradigma Neoclásico. La inclusión de dichas variables parece responder a su mera disponibilidad, ya que no se aporta ninguna teoría alternativa o complementaria a la Neoclásica. En este sentido se trata de una metodología inductiva ${ }^{9}\left(\mathrm{mi}^{-}\right.$ nería de datos) que tendría por único objetivo maximizar la explicación de la varianza construyendo ecuaciones cada vez mayores, pero sin base teórica. Como ejemplo de esto tenemos el trabajo de Ramos et al. (2009), donde se llegan

\footnotetext{
9 Aunque queda inacabada al no producir ningún tipo de generalización teórica.
} 
a utilizar hasta catorce variables cuando la posición teórica de partida (neoclásica) solo explica dos de ellas (escolaridad y experiencia).

Por último tenemos los trabajos que, como los de Núñez, abandonan el eje explicativo del paradigma Neoclásico, esto es, la productividad individual, para enfocarse deliberadamente en determinantes estructurales. Pese a que estos trabajos consiguen identificar empíricamente un conjunto de factores exógenos al modelo Neoclásico, carecen de un paradigma teórico alternativo que permita aprehenderlos y articularlos en un único relato.

En este sentido consideramos que nuestra propuesta no solo supone una alternativa al modelo Neoclásico, sino que además puede explicar holísticamente los distintos factores explicativos que han sido identificados fuera de este.

\section{Metodología}

Para ejemplificar nuestra propuesta teórica estimaremos el salario de los chilenos utilizando un modelo estadístico deducido del paradigma de la Reproducción Social, usando la encuesta CASEN $^{10}$ de 2009. Dicha encuesta contiene

10 El responsable de la encuesta CASEN es el Ministerio de Planificación de Chile. En el año 2009 tuvo una muestra de 71.460 hogares y una población de 246.924 chilenos. un conjunto de variables que nos permiten conocer algunas de las condiciones de existencia de los chilenos (género, etnia ${ }^{11}$, posición social y territorial de origen), así como sus trayectorias escolares y salarios. El procedimiento utilizado fue el modelo lineal general univariante (MLGU), ya que este nos permite realizar una regresión lineal múltiple y un análisis de varianza para una variable dependiente métrica (escolaridad o salario) usando como variables independientes tanto factores (categóricas) como covariables (métricas), además de proporcionarnos las posibles interacciones existentes entre las variables independientes.

Los indicadores más débiles corresponden a la posición social de origen (clase y campo social familiar). Lamentablemente, en Chile no contamos con ningún indicador del campo social familiar. Respecto a la clase social familiar, conocemos el capital escolar de los padres (aunque desconocemos su capital cultural total), ignoramos su capital económico y respecto a sus ocupaciones, el indicador es muy pobre, sólo diferencia entre "empresario/a", "autónomo/a", "empleado/a" y "no trabajaba".

\footnotetext{
11 Sólo un 7\% de los chilenos declara pertenecer a algún pueblo indígena, por lo que la etnicidad presenta poca capacidad predictiva. También hay que señalar que no disponemos de ningún indicador racial, ya que en Chile no se recoge información sobre el fenotipo, pese a su evidente relevancia en la estratificación social del país.
}

Cuadro 3.

Indicadores de las propiedades sociales

\begin{tabular}{|c|c|c|c|}
\hline \multicolumn{2}{|c|}{ PROPIEDADES SOCIALES } & \multirow{2}{*}{$\begin{array}{l}\text { INDICADORES } \\
\text { (CASEN 2009) } \\
\text { Sexo }\end{array}$} & \multirow{2}{*}{$\begin{array}{l}\text { MEDIDA } \\
\text { Categórica }\end{array}$} \\
\hline \multirow{8}{*}{ 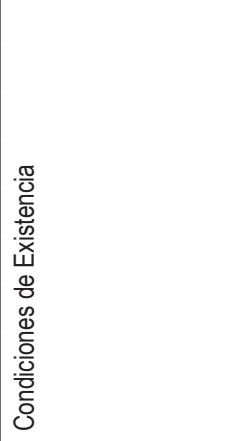 } & Género & & \\
\hline & Etnia/raza & Etnia & Categórica \\
\hline & \multirow[t]{2}{*}{ Posición Territorial de Origen } & Zona (urbano/rural) & Categórica \\
\hline & & Región & Categórica \\
\hline & \multirow[t]{5}{*}{ Posición Social de Origen } & Educación Padre & Categórica \\
\hline & & Educación Madre & Categórica \\
\hline & & Trabajo Padre & Categórica \\
\hline & & Trabajo Madre & Categórica \\
\hline \multirow{3}{*}{\multicolumn{2}{|c|}{ Trayectoria Escolar }} & & \\
\hline & & Escolaridad & Métrica \\
\hline & & Nivel Educativo & Categórica \\
\hline \multicolumn{2}{|l|}{ Experiencia Laboral } & Edad & Métrica \\
\hline \multicolumn{2}{|l|}{ Salario } & Ingreso del Trabajo & Métrica \\
\hline
\end{tabular}


Como vimos en el marco teórico, el modelo que proponemos está compuesto por dos momentos analíticos diferenciados que tratan de aprehender los elementos biográficos más relevantes para la explicación del salario; estos son, las condiciones de existencia y la trayectoria escolar.

Por tanto, en un primer momento se explicará la trayectoria escolar de los asalariados a partir de sus condiciones de existencia, y en un segundo momento, se explica el salario a partir de la interacción entre las condiciones de existencia y la trayectoria escolar.

Modelo Reproductivo:

1. Condiciones de Existencia $\rightarrow$ Trayectoria escolar

2. (Condiciones de Existencia * Trayectoria Escolar) $\rightarrow$ Salario

\section{Resultados}

En primer lugar estimaremos el salario de los chilenos ${ }^{12}$ a partir del modelo Neoclásico. Para esto utilizaremos la ecuación de Mincer, primero en su formulación original y posteriormente en una más compleja. En la primera ecuación se explica el salario mediante la educación (medida como años de escolaridad) y la experiencia laboral (indicada con la edad y el cuadrado de la edad).

Como podemos observar (tabla 1), la regresión nos indica que el $23,4 \%$ de la diferencia salarial existente entre los chilenos puede ser explicada por este modelo. Las tres variables independientes introducidas son significativas, y como vemos en los coeficientes estandarizados, la escolaridad es la variable más importe. El cuadrado de la edad presenta un coeficiente negativo, lo que nos indica que, como propuso Mincer, la edad tiene una relación cóncava con el salario.

A continuación utilizamos una ecuación de Mincer más sensible que la anterior, que nos permite capturar el efecto titulación que propone la Teoría de la Señalización. Para

12 Para que el salario de los chilenos sea comparable seleccionamos a los que tenían una jornada laboral completa, situación en la que se encontraban el $83,2 \%$ de los trabajadores. Aquellos que tenían una jornada laboral parcial $(11,5 \%)$ o prolongada $(5,3 \%)$ fueron excluidos de las estimaciones salariales. ello realizamos un MLGU reemplazando la variable continua escolaridad por la variable categórica nivel educativo, ya que esta nos permite conocer si se completó o no un determinado nivel de estudios. Además, utilizamos un resultado saturado, es decir, que contenga todos los efectos de interacción entre las variables independientes.

En la tabla 2 podemos apreciar cómo los cambios introducidos aumentan el nivel de sensibilidad del modelo Neoclásico, que pasa a explicar el $28 \%$ del salario. Estos resultados ratifican, como afirma la Teoría de la Señalización, la importancia de diferenciar entre las personas que consiguieron completar un determinado nivel educativo y las que no. Por otro lado, también comprobamos la relevancia de capturar los efectos que producen las interacciones entre las variables independientes.

A continuación, estimamos el salario de los chilenos a partir del modelo Reproductivo que proponemos. Para ello necesitamos explicar, en primer lugar, la trayectoria escolar de los actuales asalariados y posteriormente su salario. Realizaremos primero un MLGU donde las condiciones de existencia de los asalariados, esto es, su género, etnia y su posición social y territorial de origen, expliquen su trayectoria escolar (medida como años de escolaridad).

Como podemos advertir en la tabla 3, las condiciones de existencia que conseguimos capturar en nuestro modeIo, explican el $36,2 \%$ de las trayectorias escolares con el modelo de efectos principales (sin interacción entre las variables independientes) y llega al $38 \%$ con el modelo saturado $^{13}$ (con interacción entre las variables independientes). Como vemos, todas las variables son significativas y si nos fijamos en el estadístico Eta ${ }^{2}$ parcial podemos conocer la importancia de cada variable para el modelo. Observamos así que, entre las distintas condiciones de existencia, el capital cultural de los padres es la más importante, muy especialmente el de la madre, para explicar la trayectoria escolar. Le siguen en importancia la posición territorial de

13 Debido a su gran tamaño, las tablas de los modelos saturados (factorial completo) no han podido ser incluidas el artículo. Por ello, sólo presentamos las tablas de los modelos de efectos principales, pero indicando el $\mathrm{R}^{2}$ corregido de los dos modelos. Las tablas de los modelos saturados están a disposición del lector que las solicite.

Tabla 1.

Modelo Neoclásico para el Salario (Regresión)

\begin{tabular}{|c|c|c|c|c|c|c|}
\hline \multicolumn{2}{|c|}{ Modelo } & \multicolumn{2}{|c|}{ Coeficientes no estandarizados } & \multirow{2}{*}{$\begin{array}{c}\text { Coeficientes estandarizados } \\
\text { Beta }\end{array}$} & \multirow{2}{*}{$\begin{array}{l}\mathrm{t} \\
\mathrm{B}\end{array}$} & \multirow{2}{*}{$\begin{array}{l}\text { Sig. } \\
\text { Error típ. }\end{array}$} \\
\hline & & B & Error típ. & & & \\
\hline \multirow[t]{4}{*}{1} & (Constante) & $-676133,992$ & 2007,044 & & $-336,881$ &, 000 \\
\hline & Escolaridad & 59489,607 & 55,057 &, 480 & 1080,507 &, 000 \\
\hline & Edad & 11609,596 & 98,157 &, 303 & 118,276 &, 000 \\
\hline & Edad2 & $-40,057$ & 1,182 &,- 087 & $-33,892$ &, 000 \\
\hline
\end{tabular}

$\mathrm{R}$ cuadrado $=, 234$ ( $\mathrm{R}$ cuadrado corregida $=, 234)$ 
Tabla 2.

Modelo Neoclásico para el Salario (MLGU)

Pruebas de los efectos inter-sujetos

\begin{tabular}{|c|c|c|c|c|c|c|}
\hline Origen & $\begin{array}{c}\text { Suma de cuadrados } \\
\text { tipo III }\end{array}$ & gl & Media cuadrática & $\mathbf{F}$ & Sig. & $\begin{array}{c}\text { Eta al cuadrado } \\
\text { parcial }\end{array}$ \\
\hline Modelo corregido & $2,609 \mathrm{E} 17$ & 35 & $7,454 \mathrm{E} 15$ & 45877,700 &, 000 & ,280 \\
\hline Intersección & $9,517 \mathrm{E} 13$ & 1 & $9,517 \mathrm{E} 13$ & 585,753 &, 000 &, 000 \\
\hline EDUCACIÓN & 1,779E15 & 8 & $2,223 \mathrm{E} 14$ & 1368,432 &, 000 &, 003 \\
\hline EDAD & 4,229E14 & 1 & $4,229 \mathrm{E} 14$ & 2602,815 &, 000 &, 001 \\
\hline EDAD2 & 2,203E14 & 1 & 2,203E14 & 1355,883 &, 000 &, 000 \\
\hline EDUCACIÓN * EDAD & 2,244E15 & 8 & $2,805 \mathrm{E} 14$ & 1726,598 &, 000 &, 003 \\
\hline EDUCACIÓN * EDAD2 & $1,785 \mathrm{E} 15$ & 8 & 2,231E14 & 1373,370 &, 000 &, 003 \\
\hline EDAD * EDAD2 & $1,446 \mathrm{E} 14$ & 1 & $1,446 \mathrm{E} 14$ & 889,814 &, 000 &, 000 \\
\hline EDUCACIÓN * EDAD * EDAD2 & $1,735 \mathrm{E} 15$ & 8 & $2,169 \mathrm{E} 14$ & 1335,134 &, 000 & ,003 \\
\hline Error & $6,721 \mathrm{E} 17$ & 4136309 & $1,625 \mathrm{E} 11$ & & & \\
\hline Total & 1,609E18 & 4136345 & & & & \\
\hline Total corregida & $9,329 \mathrm{E} 17$ & 4136344 & & & & \\
\hline
\end{tabular}

$\mathrm{R}$ cuadrado $=, 280$ ( $\mathrm{R}$ cuadrado corregida $=, 280$ )

Tabla 3.

Condiciones de Existencia como determinantes de la Trayectoria Escolar (MLGU)

Pruebas de los efectos inter-sujetos

\begin{tabular}{|c|c|c|c|c|c|c|}
\hline Origen & $\begin{array}{c}\text { Suma de cuadrados } \\
\text { tipo III }\end{array}$ & gl & Media cuadrática & $F$ & Sig. & $\begin{array}{c}\text { Eta al cuadrado } \\
\text { parcial }\end{array}$ \\
\hline Modelo corregido & $40321392,865^{a}$ & 31 & 1300690,092 & 107663,996 &, 000 & ,362 \\
\hline Intersección & 32307954,099 & 1 & 32307954,099 & 2674275,348 &, 000 & ,312 \\
\hline SEXO & 249793,836 & 1 & 249793,836 & 20676,565 &, 000 & ,003 \\
\hline REGIÓN & 322778,233 & 14 & 23055,588 & 1908,415 &, 000 & ,005 \\
\hline ZONA & 1416146,517 & 1 & 1416146,517 & 117220,846 &, 000 & ,020 \\
\hline ETNIA & 10465,054 & 1 & 10465,054 & 866,240 &, 000 &, 000 \\
\hline TRABAJOPADRE & 97257,109 & 3 & 32419,036 & 2683,470 &, 000 &, 001 \\
\hline TRABAJOMADRE & 19656,635 & 3 & 6552,212 & 542,356 &, 000 &, 000 \\
\hline EDUCAPADRE & 3138536,154 & 4 & 784634,038 & 64947,705 &, 000 & ,042 \\
\hline EDUCAMADRE & 5975074,123 & 4 & 1493768,531 & 123645,971 &, 000 & ,077 \\
\hline Error & 71191691,573 & 5892858 & 12,081 & & & \\
\hline Total & $7,666 \mathrm{E} 8$ & 5892890 & & & & \\
\hline Total corregida & $1,115 \mathrm{E} 8$ & 5892889 & & & & \\
\hline
\end{tabular}

origen y el género. La pertenecía étnica, como ya habíamos advertido, es la propiedad menos importante pese a ser significativa.

El segundo momento de nuestro modelo consiste en explicar el salario mediante la interacción entre las condiciones de existencia y la trayectoria escolar. En este caso excluiremos la posición territorial de origen (zona y región), ya que estas variables podrían capturar efectos salariales relacionados con la demanda del trabajo (como el tamaño de las empresas o la estructura productiva) y nuestro análisis se quiere centrar netamente en la oferta de trabajo (las propiedades de los asalariados). Hemos tomado la decisión de excluir también de nuestro modelo la experiencia laboral (edad y el cuadrado de la edad) renunciando así a su probada capacidad explicativa. Lo hacemos para ser consecuentes con nuestra posición teórica, ya que esta propiedad social (la experiencia laboral) no ha sido teorizada desde el paradigma de la Reproducción Social ${ }^{14}$.

Para indicar la trayectoria escolar utilizaremos las variables escolaridad (años) y el cuadrado de la escolaridad. El objetivo de esta última variable es el de incluir la hipótesis

14 En cualquier caso consideramos que el efecto de la experiencia laboral sobre el salario podría ser explicado desde la teoría de los campos sociales. La permanencia en un campo permite justamente la acumulación del tipo de capital que determina la posición de los agentes en él. 
Tabla 4.

Condiciones de Existencia y Trayectoria Escolar como determinantes del Salario (MLGU)

Pruebas de los efectos inter-sujetos

\begin{tabular}{|c|c|c|c|c|c|c|}
\hline Origen & $\begin{array}{c}\text { Suma de cuadrados } \\
\text { tipo III }\end{array}$ & gl & Media cuadrática & $F$ & Sig. & $\begin{array}{c}\text { Eta al cuadrado } \\
\text { parcial }\end{array}$ \\
\hline Modelo corregido & $2,716 \mathrm{E} 17$ & 18 & $1,509 \mathrm{E} 16$ & 72007,069 & ,000 & ,385 \\
\hline Intersección & $1,273 \mathrm{E} 16$ & 1 & $1,273 \mathrm{E} 16$ & 60735,592 &, 000 & ,029 \\
\hline SEXO & $2,303 E 16$ & 1 & $2,303 E 16$ & 109881,807 &, 000 & ,050 \\
\hline ETNIA & $2,138 \mathrm{E} 14$ & 1 & $2,138 \mathrm{E} 14$ & 1020,182 &, 000 &, 000 \\
\hline TRABAJOPADRE & $2,926 \mathrm{E} 15$ & 3 & $9,754 \mathrm{E} 14$ & 4654,396 &, 000 & ,007 \\
\hline TRABAJOMADRE & $7,982 \mathrm{E} 14$ & 3 & 2,661E14 & 1269,647 &, 000 & ,002 \\
\hline EDUCAPADRE & $5,483 \mathrm{E} 15$ & 4 & 1,371E15 & 6540,985 &, 000 & ,012 \\
\hline EDUCAMADRE & 2,591E15 & 4 & $6,477 \mathrm{E} 14$ & 3090,499 &, 000 &, 006 \\
\hline ESCOLARIDAD & 1,947E16 & 1 & 1,947E16 & 92887,517 &, 000 &, 043 \\
\hline ESCOLARIADAD ${ }^{2}$ & $5,169 \mathrm{E} 16$ & 1 & $5,169 \mathrm{E} 16$ & 246666,288 &, 000 & , 107 \\
\hline Error & $4,336 \mathrm{E} 17$ & 2068970 & $2,096 \mathrm{E} 11$ & & & \\
\hline Total & 1,189E18 & 2068989 & & & & \\
\hline Total corregida & $7,052 \mathrm{E} 17$ & 2068988 & & & & \\
\hline
\end{tabular}

Efectos Principales: $\mathrm{R}$ cuadrado $=, 385$ ( $\mathrm{R}$ cuadrado corregida $=, 385)$

Factorial Completo: $\mathrm{R}$ cuadrado $=, 584(\mathrm{R}$ cuadrado corregida $=, 583)$

bourdieuniana de que la inversión en capital escolar solo muestra su auténtica rentabilidad en los niveles más altos, aquellos a los que solo pueden acceder las clases superiores. Si esto es así, la relación entre la escolaridad y el salario debería ser convexa y no lineal.

En la tabla 4 podemos observar que el modelo explica el $38,5 \%$ de la diferencia salarial con el análisis de efectos principales y el $58,3 \%$ con el análisis saturado. Advertimos también que todas las variables introducidas son significativas. Respecto a la importancia de las propiedades sociales, observamos que la trayectoria escolar es la más importante, especialmente la variable escolaridad al cuadrado, seguida por el género y la clase social de origen. Dentro de esta cobran relevancia las propiedades del padre sobre las de la madre. El grupo étnico vuelve a ser la propiedad menos relevante pese a ser significativa.

Si nos fijamos en las estimaciones de los parámetros (tabla 5) podemos comprobar que el beta de la variable escolaridad es negativo mientras que, como se esperaba, el del cuadrado de la escolaridad es positivo. También observamos que el efecto de la segunda (Eta ${ }^{2}$ parcial: 0,107$)$ es más del doble que el de la primera (Eta ${ }^{2}$ parcial: 0,043 ). Esto nos señala que, efectivamente, la relación entre la escolaridad y el salario no es lineal sino convexa. Supone que la escolaridad solo afecta significativamente al salario a partir de superar cierto umbral que, una vez es traspasado, aumenta exponencialmente el salario. Es decir, existe un premio salarial desproporcionado para el nivel más alto de escolaridad.

Siguiendo con el estadístico beta, también podemos apreciar cómo los hombres reciben un salario superior al de las mujeres y cómo los asalariados que pertenecen a grupos indígenas reciben salarios inferiores a los que no pertenecen. Respecto a la clase social de origen, observamos cómo los hijos de empresarios obtienen salarios más altos que los hijos de autónomos, trabajadores/empleados o padres que no trabajaban. En el caso de las madres, son las que no trabajaban las que están asociadas a hijos con salarios más altos, mientras que tener una madre empresaria afecta negativamente al salario ${ }^{15}$. También comprobamos que los salarios más altos están asociados a tener padres universitarios ${ }^{16}$.

Llegados a este punto y vista la importancia que tienen las condiciones de existencia tanto para determinar la trayectoria escolar como para determinar los salarios, cabe preguntarse qué capacidad tendrá para explicar por sí sola (sin la trayectoria escolar) los salarios. Esto es, nos preguntamos en qué medida podemos conocer el salario de los chilenos antes de que éstos ingresen al sistema escolar, o si prefiere, nada más nacer. Para conocer esto realizaremos un MLGU utilizando exclusivamente las variables que indican las condiciones de existencia en las que nacieron los asalariados.

15 Esto podría indicar que la mayoría de las empresarias chilenas tiene una situación muy precaria, estando situadas socialmente por debajo de las trabajadoras por cuenta ajena y muy lejos de las que "no necesitan trabajar".

16 El nivel educativo de los padres es el siguiente: 1 = Sin estudios, 2 = Básicos, 3 = Medios, 4 = Técnicos Superiores y 5 = Universitarios. Esta última (universitarios) es la categoría de referencia en el análisis. 
Tabla 5.

Condiciones de Existencia y Trayectoria Escolar como determinantes del Salario (MLGU)

\begin{tabular}{|c|c|c|c|c|c|c|c|}
\hline \multirow[b]{2}{*}{ Parámetro } & \multirow[b]{2}{*}{ B } & \multirow[b]{2}{*}{ Error típ. } & \multirow[b]{2}{*}{$\mathrm{t}$} & \multirow[b]{2}{*}{ Sig. } & \multicolumn{2}{|c|}{ Intervalo de confianza 95\% } & \multirow{2}{*}{$\begin{array}{c}\text { Eta al cuadrado } \\
\text { parcial }\end{array}$} \\
\hline & & & & & Límite inferior & Límite superior & \\
\hline Intersección & 830730,881 & 4255,924 & 195,194 &, 000 & 822389,421 & 839072,340 & ,018 \\
\hline [SEXO= HOMBRE] & 215532,060 & 650,203 & 331,484 &, 000 & 214257,686 & 216806,435 & ,050 \\
\hline [SEXO=MUJER] & $0^{\mathrm{a}}$ & & & $\cdot$ & & . & · \\
\hline [ETNIA= INDÍGENA] & $-44988,148$ & 1408,508 & $-31,940$ &, 000 & $-47748,774$ & $-42227,523$ & ,000 \\
\hline [ETNIA=NO INDÍGENA] & $0^{\mathrm{a}}$ & & & . & & . & . \\
\hline [TRABAJOPADRE= EMPRESARIO] & 167559,519 & 3780,434 & 44,323 &, 000 & 160150,002 & 174969,036 & ,001 \\
\hline [TRABAJOPADRE= AUTÓNOMO] & $-2805,684$ & 3411,062 &,- 823 & ,411 & $-9491,245$ & 3879,876 & ,000 \\
\hline [TRABAJOPADRE= TRABAJADOR] & $-39699,459$ & 3360,255 & $-11,814$ &, 000 & $-46285,440$ & $-33113,479$ &, 000 \\
\hline [TRABAJOPADRE= NO TRABAJABA] & $0^{\mathrm{a}}$ & & & 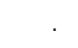 & & . & . \\
\hline [TRABAJOMADRE= EMPRESARIA] & $-181536,548$ & 3456,090 & $-52,527$ &, 000 & $-188310,362$ & $-174762,734$ & ,001 \\
\hline [TRABAJOMADRE= AUTÓNOMA] & $-35174,506$ & 1283,555 & $-27,404$ &, 000 & $-37690,228$ & $-32658,783$ &, 000 \\
\hline [TRABAJOMADRE= TRABAJADORA] & $-21500,190$ & 759,506 & $-28,308$ &, 000 & $-22988,795$ & $-20011,585$ &, 000 \\
\hline [TRABAJOMADRE= NO TRABAJABA] & $0^{\mathrm{a}}$ & & & . & . & . & . \\
\hline [EDUCAPADRE=1] & $-229667,619$ & 1991,615 & $-115,317$ &, 000 & $-233571,114$ & $-225764,124$ & ,006 \\
\hline [EDUCAPADRE=2] & $-246370,139$ & 1640,247 & $-150,203$ &, 000 & $-249584,964$ & $-243155,314$ & 011 \\
\hline [EDUCAPADRE=3] & $-232057,184$ & 1510,617 & $-153,618$ &, 000 & $-235017,939$ & $-229096,429$ &, 011 \\
\hline [EDUCAPADRE=4] & $-170159,800$ & 1996,724 & $-85,219$ &, 000 & $-174073,308$ & $-166246,291$ & ,003 \\
\hline [EDUCAPADRE=5] & $0^{\mathrm{a}}$ & & & . & & & \\
\hline [EDUCAMADRE=1] & $-221843,217$ & 2243,507 & $-98,882$ &, 000 & $-226240,410$ & $-217446,023$ & ,005 \\
\hline [EDUCAMADRE=2] & $-195427,903$ & 1935,454 & $-100,973$ &, 000 & $-199221,325$ & $-191634,482$ & ,005 \\
\hline [EDUCAMADRE=3] & $-159263,162$ & 1811,460 & $-87,920$ &, 000 & $-162813,560$ & $-155712,764$ & ,004 \\
\hline [EDUCAMADRE=4] & $-48950,558$ & 2880,468 & $-16,994$ &, 000 & $-54596,172$ & $-43304,943$ & ,000 \\
\hline [EDUCAMADRE=5] & $0^{\mathrm{a}}$ & & & . & & . & . \\
\hline ESCOLARIDAD & $-106164,992$ & 348,339 & $-304,775$ &, 000 & $-106847,725$ & $-105482,260$ & ,043 \\
\hline ESCOLARIADAD² & 7758,801 & 15,622 & 496,655 &, 000 & 7728,183 & 7789,420 & 107 \\
\hline
\end{tabular}

Como podemos ver en la tabla 6 , las condiciones de existencia en las que nacieron los chilenos explican el $20,3 \%$ de sus diferencias salariales con el análisis de efectos principales y el $31,8 \%$ con el análisis saturado. Es destacable que las condiciones de existencia tengan mayor capacidad explicativa que el modelo Neoclásico (28\%), teniendo en cuenta que vienen dadas desde la infancia, estando biográficamente muy alejadas del momento laboral. El modelo Neoclásico por su parte, utiliza variables contiguas al momento laboral (educación) o simultáneas a él (experiencia laboral).

Finalmente, nos interesa conocer cómo varía la capacidad explicativa de los modelos presentados entre las distintas generaciones de chilenos que actualmente componen el mercado laboral. Hay que recordar que hasta ahora hemos analizado al conjunto de la población asalariada sin distinción etaria alguna. Este tipo de análisis es interesante ya que, debido a los cambios significativos ocurridos en el acceso a la educación, podría identificar a generaciones que respondan a distintos patrones, así como cambios de tendencia en los determinantes del salario en Chile. Para llevarlo a cabo, hemos segmentado la muestra por cohortes de edad generando cuatro grupos etarios: Ios asalariados de 25 a 35 años, los de 36 a 45 años, los de 46 a 55 años y los de 56 a 65 años. El primer grupo pertenece a la generación de chilenos que nacieron entre 1974 y 1984, el segundo entre 1964 y 1973, el tercero entre 1954 y 1963 y el último grupo entre 1944 y 1953. Repetimos todos los análisis anteriores para cada una de las generaciones, los resultados se muestran a continuación (tabla 7 y gráfico 5).

Como podemos apreciar, el modelo Reproductivo es, con diferencia, el que tiene mayor capacidad explicativa para todos los grupos etarios. Además es el modelo más estable, oscilando entre un máximo del $72,4 \%$ y un mínimo del $65 \%$ del salario explicado. Frente a él, el modelo Neoclásico oscila entre un máximo de 30,4\% y un mínimo del 19,3\%. Observamos también, que las condiciones de existencia de los chilenos siguen teniendo una mayor capacidad explicativa que el modelo Neoclásico en todos los cohortes de edad, oscilando entre un máximo del $51,6 \%$ y un mínimo del $41,3 \%$. 
Tabla 6.

Condiciones de Existencia como determinantes del Salari $O$ (MLGU)

Pruebas de los efectos inter-sujetos

\begin{tabular}{|c|c|c|c|c|c|c|}
\hline Origen & $\begin{array}{c}\text { Suma de cuadrados } \\
\text { tipo III }\end{array}$ & gl & Media cuadrática & $\mathrm{F}$ & Sig. & $\begin{array}{c}\text { Eta al cuadrado } \\
\text { parcial }\end{array}$ \\
\hline Modelo corregido & $1,429 \mathrm{E} 17$ & 16 & $8,932 \mathrm{E} 15$ & 32864,964 &, 000 & ,203 \\
\hline Intersección & $4,626 \mathrm{E} 16$ & 1 & $4,626 \mathrm{E} 16$ & 170210,327 &, 000 & ,076 \\
\hline SEXO & $1,678 \mathrm{E} 16$ & 1 & $1,678 \mathrm{E} 16$ & 61750,673 &, 000 &, 029 \\
\hline TRABAJOPADRE & 5,691E15 & 3 & 1,897E15 & 6980,007 &, 000 & ,010 \\
\hline TRABAJOMADRE & $5,239 \mathrm{E} 14$ & 3 & $1,746 \mathrm{E} 14$ & 642,549 &, 000 & 001 \\
\hline EDUCAPADRE & $2,400 \mathrm{E} 16$ & 4 & $5,999 \mathrm{E} 15$ & 22072,894 &, 000 & 041 \\
\hline EDUCAMADRE & $1,988 \mathrm{E} 16$ & 4 & $4,969 \mathrm{E} 15$ & 18283,430 &, 000 & ,034 \\
\hline ETNIA & $6,418 \mathrm{E} 14$ & 1 & $6,418 \mathrm{E} 14$ & 2361,566 &, 000 & ,001 \\
\hline Error & $5,623 \mathrm{E} 17$ & 2068972 & $2,718 \mathrm{E} 11$ & & & \\
\hline Total & 1,189E18 & 2068989 & & & & \\
\hline Total corregida & $7,052 \mathrm{E} 17$ & 2068988 & & & & \\
\hline
\end{tabular}

Tabla 7.

Determinantes del Salario por Cohortes de edad

\begin{tabular}{cccc}
\hline Cohortes de edad & $\begin{array}{c}\text { Modelo } \\
\text { Neoclásico }\end{array}$ & $\begin{array}{c}\text { Modelo } \\
\text { Reproductivo }\end{array}$ & $\begin{array}{c}\text { Condiciones de } \\
\text { Existencia }\end{array}$ \\
\hline $25-35$ & 24,6 & 65 & 42,7 \\
$36-45$ & 19,3 & 72,4 & 51,6 \\
$46-55$ & 23,2 & 70,8 & 42,9 \\
$56-65$ & 30,4 & 69,2 & 41,3 \\
Población Total & 28 & 58,4 & 31,8 \\
\hline
\end{tabular}

R cuadrado: Modelo Factorial Completo

Comparando intergeneracionalmente los determinantes del salario, podemos apreciar cómo el modelo Reproductivo aumenta su capacidad explicativa de manera constante salvo en la generación más joven, donde disminuye ligeramente. De manera inversa, el modelo Neoclásico la reduce constantemente entre estas cohortes. La tendencia de ambos modelos cambia al pasar de la cohorte de 36 a 45 años a la más joven, dónde disminuye la capacidad del modelo Reproductivo y aumenta la del Neoclásico.

Creemos que las diferencias que apreciamos entre grupos etarios podrían deberse fundamentalmente a dos factores. Por un lado podrían estar indicando la evolución histórica que han tenido los determinantes del salario en Chile, que habrían cambiado como consecuencia de la transformación de la estructura económica y social del país, así como por la acción del Estado. Siguiendo esta línea tendríamos que interpretar nuestros resultados afirmando que en Chile, la reproducción social del salario ha venido aumentado sistemáticamente hasta hace una década, momento en el que empezó a declinar. Por otro lado, nuestros resultados

Gráfico 5.

Determinantes del Salario por Cohortes de edad
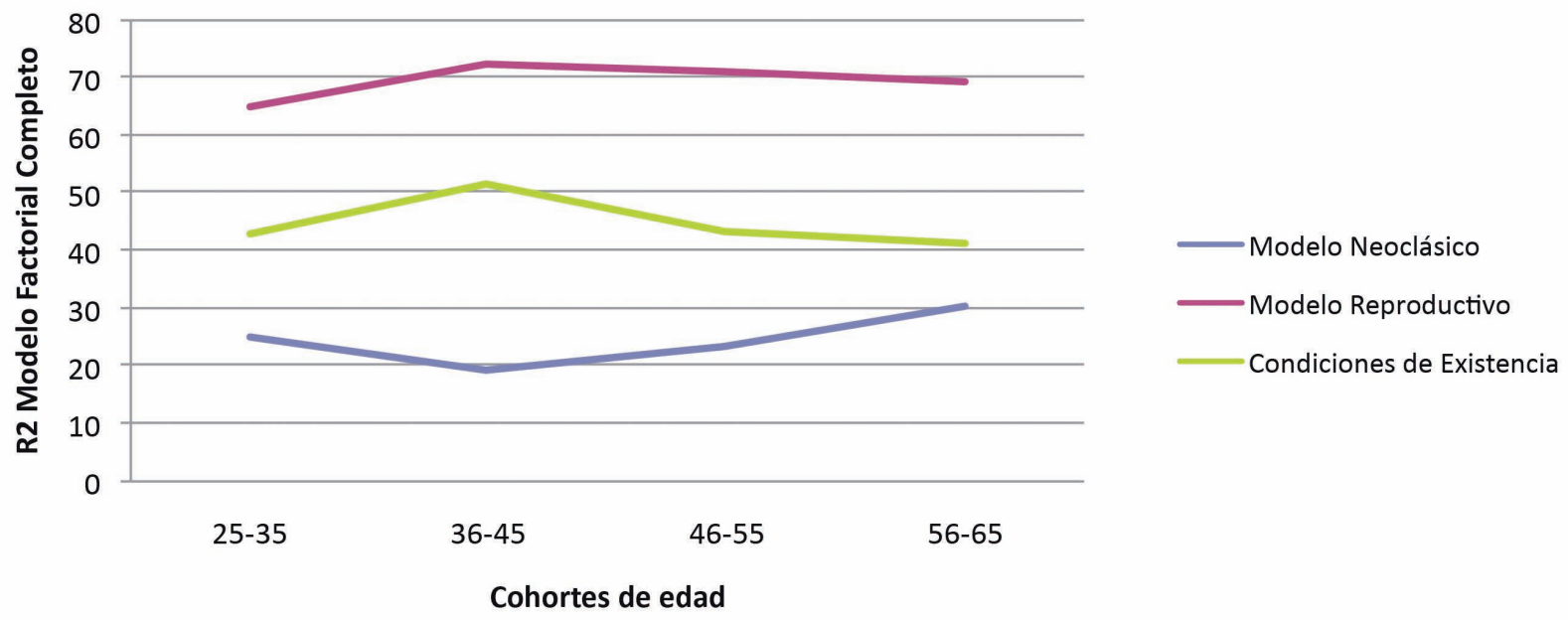

RIS [online] 2015, 73 (3), e019. REVISTA INTERNACIONAL DE SOCIOLOGÍA. ISSN-L: 0034-9712 
podrían estar indicándonos cómo cambian los determinantes del salario a lo largo del ciclo laboral de los chilenos. Desde esta perspectiva podríamos decir que los efectos de la reproducción social aumentan cuando los asalariados se encuentran entre los 36 y 45 años, reduciéndose (muy levemente) desde ese momento hasta el final de su ciclo laboral. Lo cierto es que sólo un análisis diacrónico podría revelar cuál de estas dos interpretaciones es la adecuada ${ }^{17}$.

\section{ConCLUSIONES}

En la primera parte del trabajo se han presentado los supuestos teóricos mediante los que el paradigma Neoclásico, hegemónico en Economía, explica e interpreta las diferencias salariales derivadas de la oferta de trabajo (los asalariados). Frente a este, se han presentado los supuestos teóricos del paradigma sociológico de la Reproducción Social, utilizando para ello parte de la obra de Bourdieu. Ambos paradigmas teóricos se diferencia en cuatro aspectos: I) Su objeto/ sujeto de análisis; el individuo abstracto para el paradigma Neoclásico frente a los grupos sociohistóricos para el de la Reproducción Social. II) Su posición epistemológica; particularista para el paradigma Neoclásico y holística para el de la Reproducción Social. III) Su eje explicativo; la productividad individual para el paradigma Neoclásico y la herencia social para el paradigma de la Reproducción Social. Y V) su posición política; conservadora para el paradigma Neoclásico y crítica para el de la Reproducción Social. Además, se ha criticado la falta de realismo de algunos supuestos neoclásicos, especialmente la explicación del nivel educativo. Por lo anterior, se concluye que el paradigma Neoclásico es inadecuado para el caso chileno, tanto por sus problemas teóricos (supuestos irreales) como por sus consecuencias políticas (legitimación de la desigualdad económica).

En la segunda parte del trabajo, para ejemplificar nuestra propuesta teórica y conocer su grado de adecuación al caso chileno, hemos estimado empíricamente el salario utilizando dos modelos estadísticos, primero un modelo Neoclásico (tipo Mincer) y posteriormente un modelo Reproductivo que trata de capturar los factores explicativos de nuestra propuesta teórica. Los resultados nos muestran que el modelo Reproductivo tiene mayor capacidad explicativa que el Neoclásico, tanto para la población asalariada en general, como para cada una de las generaciones que la componen. También hemos podido comprobar cómo las condiciones de existencia de los chilenos explican por sí solas más diferencia salarial que el modelo Neoclásico, pese a que estas vienen determinadas desde la infancia

\footnotetext{
17 Lamentablemente, las variables necesarias para realizar este trabajo sólo están disponibles en la encuesta CASEN 2006, por lo que un estudio diacrónico sólo habría tenido dos puntos de referencia (2006 y 2009) con una distancia irrelevante (3 años).
}

y el modelo Neoclásico (también el Reproductivo) requiere conocer la trayectoria educativa de los asalariados. Por último hay que señalar que nuestros resultados apoyan la hipótesis (reproductiva) de que la relación entre el nivel educativo y el salario no es lineal, sino convexa (función cuadrática). Es decir, que el premio salarial para los últimos años de escolaridad es mucho mayor que para los anteriores. Por lo anterior, se concluye que el modelo Reproductivo es adecuado para dar cuenta de las diferencias salariales en Chile.

Lejos de su discurso meritocrático, comprobamos que el modelo neoliberal chileno no asigna los salarios a partir de la capacidad individual de los asalariados, sino que lo hace, fundamentalmente, a partir de sus propiedades sociales. Todo indica que la jibarización estatal que sufrió el país no aumentó la meritocracia salarial, sino que contrariamente, facilitó la reproducción social de sus clases privilegiadas. Tampoco parece que el aumento educativo que han experimentado las generaciones más jóvenes esté acortando el alto nivel de desigualdad económica. En este sentido consideramos, que sólo la reaparición de un Estado Social redistribuidor podría reducir significativamente los niveles de desigualdad y de reproducción social.

Para mejorar la sensibilidad empírica del modelo Reproductivo sería muy importante contar con un indicador racial, puesto que en Chile, al igual que en el resto de América, el fenotipo es un factor determinante de la estructura social del país. Habría que contar también con un indicador del campo social familiar para conocer su efecto sobre la trayectoria social (escolar y laboral) de los hijos. Y por último deberíamos tener más información sobre la clase social familiar de los asariados, especialmente sobre su capital económico y sobre la ocupación de los padres. Es probable que estos indicadores aumenten significativamente la capacidad explicativa del modelo Reproductivo permitiéndonos conocer mejor los determinantes del salario en Chile.

Aunque este estudio se ha centrado en analizar la oferta de trabajo (características de los asalariados) resulta también necesario abordar el análisis de la demanda (tamaño de empresas, sectores productivos, etc.) desde el paradigma de la Reproducción Social, así como la relación entre ambas.

Finalmente hay que interrogarse tanto por las causas del alto gasto educativo familiar, como por sus consecuencias: ¿Qué efecto ha tenido sobre el gasto educativo de las familias el discurso (oficial) del Capital Humano reproducido, desde 1980, tanto por el Estado chileno como por el marketing su mercado educativo? ¿Es posible que la inversión educativa ${ }^{18}$ que están realizando las familias chilenas, posibilitada normalmente por el endeudamiento, no consiga los retornos esperados?

\footnotetext{
18 El costo de la educación superior en Chile es, en términos relativos, el más alto del mundo (Mayol 2012).
} 


\section{BiblografíA}

Althusser, L. 1974. Ideología y aparatos ideológicos de Estado. Buenos Aires: Nueva Visión.

Arrow, K. 1973. "Higher Education as a Filter". Journal of Public Economics 2:193-216. http://dx.doi.org/10.1016/0047-2727(73)90013-3

Becker, G. 1983. El Capital Humano. Madrid: Alianza.

Bourdieu, P. 2001. Poder, Derecho y Clases Sociales. Bilbao: Desclée.

Bourdieu, P. 2003. La dominación masculina. Barcelona: Anagrama.

Bourdieu, P. 2008. Homo academicus. Madrid: Siglo XXI.

Bourdieu, P. y Passeron, J. C. 2001. La reproducción. Elementos para una teoría del sistema de enseñanza. Madrid: Popular.

Brunner, J. J. 2009a. Educación superior en Chile, Instituciones, mercados y políticas gubernamentales 1967- 2007. Santiago de Chile: Ed. Universidad Diego Portales.

Brunner, J. J. 2009b. "Apuntes sobre sociología de la educación superior en contexto internacional, regional y local". Estudios Pedagógicos 35(2): 203-230.

Camargo, R. 2007. "Del "crecimiento con equidad" al "sistema de protección social": La matriz ideológica del Chile Actual (19902007)". Revista de Sociología 21: 9-31.

Canales, M. 2007. "Ni pobres ni incluidos: ¿nueva cuestión social?" Revista de Sociología 21: 193-217.

Castillo, J. y Cabezas, G. 2010. "Caracterización de Jóvenes primera generación en Educación Superior. Nuevas Trayectoria hacia la equidad educativa". Revista Calidad en la Educación 32: 44-76.

Consejo Asesor Presidencial para la Educación Superior. 2008. Los desafíos de la educación superior. Santiago de Chile.

Encuesta CASEN. 2010. "Publicaciones" Santiago de Chile: Ministerio de Planificación. Consulta 23 de noviembre de 2012. (http:// www.mideplan.gob.cl/casen/publicaciones.html)

Estudio Económico de Chile. 2010. "Síntesis"París: Organización para la Cooperación y el Desarrollo Económico. Consulta 11 de junio del 2012 (http://www.oecd.org/dataoecd/7/38/44493040.pdf)

Garretón, M. A. y Garretón, R. 2010. "La democracia incompleta en Chile: la realidad tras los ranking internacionales". Revista de Ciencia Política 30(1): 115-148.

Lagos, M. 2007. "Chile: una prosperidad no democrática". Revista de Sociología 21: 139-163.

Marx, K. 2005. El capital, tomo III. El proceso de producción capitalista. Buenos Aires: Siglo XXI.

Marx, K. y Engels, F. 1994. La ideología alemana. Valencia: Universitat de Valencia.

Mayol, A. 2012. El derrumbe del modelo: la crisis de la economía de mercado en el Chile contemporáneo. Santiago de Chile: LOM.

Mincer, J. 1974. Schooling, Experience and Earnings. Nueva York: Columbia University Press.
Núñez, J. y Gutiérrez, R. 2004. "Class discrimination and meritocracy in the labor market: evidence from Chile". Estudios de Economía 31(2): 113-132.

Núñez, J. y Pérez, G. 2007. "'Dime cómo te llamas y te diré quién eres": La ascendencia como mecanismo de diferenciación social en Chile". Working Paper 269. Departamento de Economía de la Universidad de Chile. Santiago de Chile.

Núñez, J. y Risco, C. 2004. "Movilidad intergeneracional del ingreso en un país en desarrollo: El caso de Chile". Working Paper 210. Departamento de Economía de Universidad de Chile. Santiago de Chile.

OCDE. 2009. La Educación Superior en Chile. Serie Revisión de Políticas Nacionales de Educación. OCDE - Banco Mundial.

Perla, P. 2007. "El retorno de la teoría del capital humano". Fundamentos en Humanidades 8(2): 9-26.

Ramos, J. Rubio, C. González, M. P. y Coble, D. 2009. "Determinantes de los salarios de las carreras de Ingeniería Comercial y de Contador Auditor". Working Paper 299. Departamento de Economía de la Universidad de Chile. Santiago de Chile.

Sapelli, C. 2003. "Ecuaciones de Mincer y tasas de retorno de la educación en Chile: 1990-1998". Working Paper 254. Instituto de Economía de la Pontificia Universidad Católica de Chile. Santiago de Chile.

Sapelli, C. 2009. "Los retornos de la educación en Chile: Estimaciones por corte transversal y por cohortes". Working Paper 349. Instituto de Economía de la Pontificia Universidad Católica de Chile. Santiago de Chile.

Schultz, T. W. 1983. "La Inversión en Capital Humano". Educación y Sociedad 8(3).

Spence, M. 1973. "Job Market Signalling". Quarterly Journal of Economics 87: 355-374. http://dx.doi.org/10.2307/1882010

Stiglitz, J. 1975. "The Theory of Screening, Education and the Distribution of Income". American Economic Review, Junio: 283-300.

Thurow, L. 1983. "Educación e Igualdad Económica". Educación y Sociedad 2: 159-172.

Toharia, L. 2005. El mercado de trabajo: Teorías y aplicaciones. Madrid: Alianza.

Zapata, G. y Tejeda, I. 2008. Aseguramiento de la Calidad: Políticas Públicas y Gestión Universitaria. Proyecto ALFA nº. DCI-ALA 2008/42: 1- 64 .

LUIS MIGUEL RODRIGO BENITO es Licenciado y doctorado en Sociología por la Universidad de Valencia. Trabajo como profesor desde el 2008 en el Departamento de Economía de la Universidad Católica del Norte, Chile. Y actualmente es el Director del Instituto de Economía Aplicada Regional (IDEAR). Sus líneas de investigación son por la Sociología Económica y la Sociología Política. 